\title{
Optimization of Fixed Microphone Array in High Speed Train Noises Identification Based on Far-Field Acoustic Holography
}

\author{
Rujia Wang and Shaoyi Bei \\ School of Automotive and Transportation, Jiangsu University of Technology, Changzhou, Jiangsu, China \\ Correspondence should be addressed to Rujia Wang; rujia.wang@jsut.edu.cn
}

Received 20 July 2016; Revised 24 December 2016; Accepted 4 January 2017; Published 1 February 2017

Academic Editor: Marc Asselineau

Copyright (C) 2017 Rujia Wang and Shaoyi Bei. This is an open access article distributed under the Creative Commons Attribution License, which permits unrestricted use, distribution, and reproduction in any medium, provided the original work is properly cited.

\begin{abstract}
Acoustical holography has been widely applied for noise sources location and sound field measurement. Performance of the microphones array directly determines the sound source recognition method. Therefore, research is very important to the performance of the microphone array, its array of applications, selection, and how to design instructive. In this paper, based on acoustic holography moving sound source identification theory, the optimization method is applied in design of the microphone array, we select the main side lobe ratio and the main lobe area as the optimization objective function and then put the optimization method use in the sound source identification based on holography, and finally we designed this paper to optimize microphone array and compare the original array of equally spaced array with optimization results; by analyzing the optimization results and objectives, we get that the array can be achieved which is optimized not only to reduce the microphone but also to change objective function results, while improving the far-field acoustic holography resolving effect. Validation experiments have showed that the optimization method is suitable for high speed trains sound source identification microphone array optimization.
\end{abstract}

\section{Introduction}

The noise of high speed vehicles such as high speed trains is one of the severest noise pollution sources $[1,2]$. Based on studies in the reconstruction of the sound field microphone array, predecessors to build a grid array cross array have inherent defects; namely, in order to ensure a small main lobe width of the sound field reconstruction to improve resolution, the need to maintain a larger size of the array, such that the spacing between adjacent array elements, is increased and causes the emergence of grating lobes, which greatly weakened the ability of the sound field reconstruction array.

Compared with beamforming, acoustic holography method can achieve a quantitative measure of moving sound source, so in recent years it has been widely studied and applied. Acoustic holography theory in the 1980 s by the Williams and Maynard et al. [3-5] put forward the strict acoustic radiation based on the theory. In the 1990s, Tanaka et al. [6] were first used the far-field acoustic holography method to measure a vehicle noise source and in 2004 by using two-dimensional arrays based on far-field acoustic holography method of analyzing tire/road noise and tire noise successfully. From 1998 to 2008, Park et al. $[7,8]$ established a framework for holographic mobile, using sound field space transformation method to eliminate the Doppler effect and measure and analyze a low-speed train noise. Yang et al. [9-11] first proposed the international far-field diffraction acoustic holography method and in 2010 proposed eliminating the time-domain method Doppler effect, for the first time to achieve a speed of $117 \mathrm{~km} / \mathrm{h}$, vehicle quantitative identification of noise sources outside the vehicle.

In this paper, based on formation simulate annealing optimization method to optimize the unequal a fixed spacing array, rather than a random array, for obtaining a more accurate result of the sound source identification, it can 


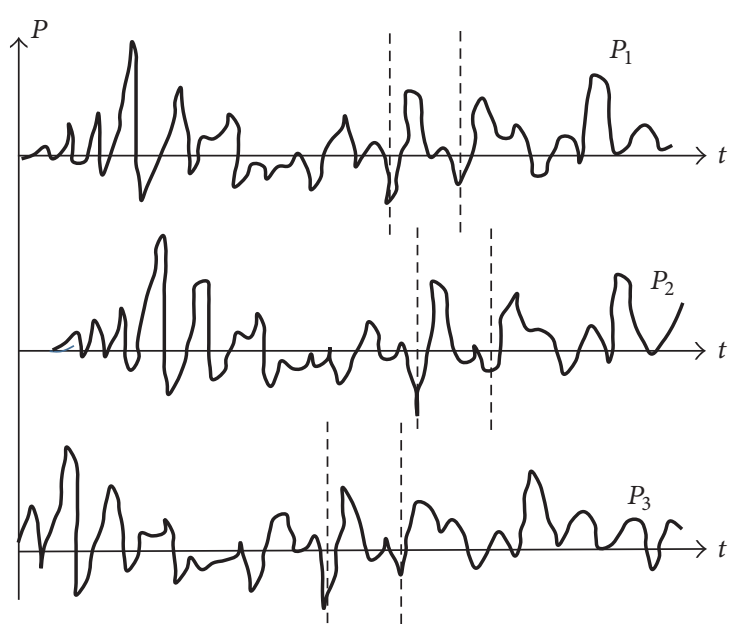

(a)

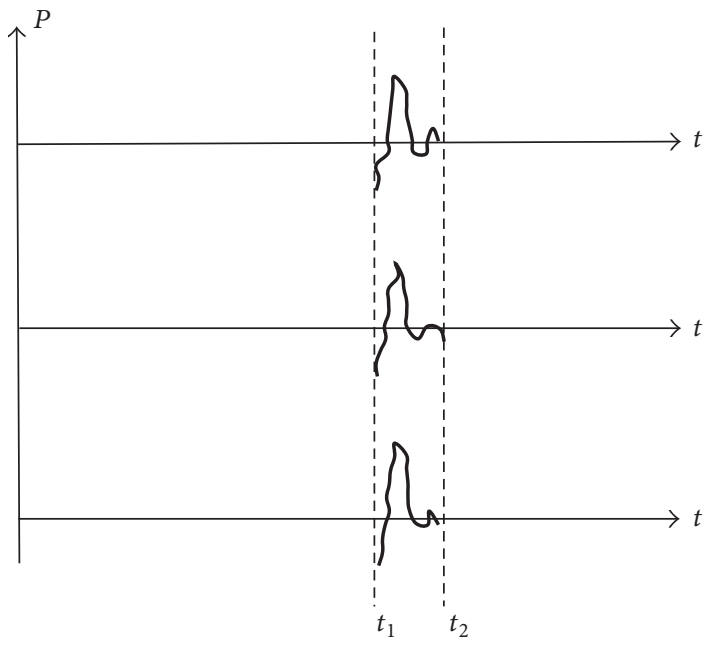

(c)

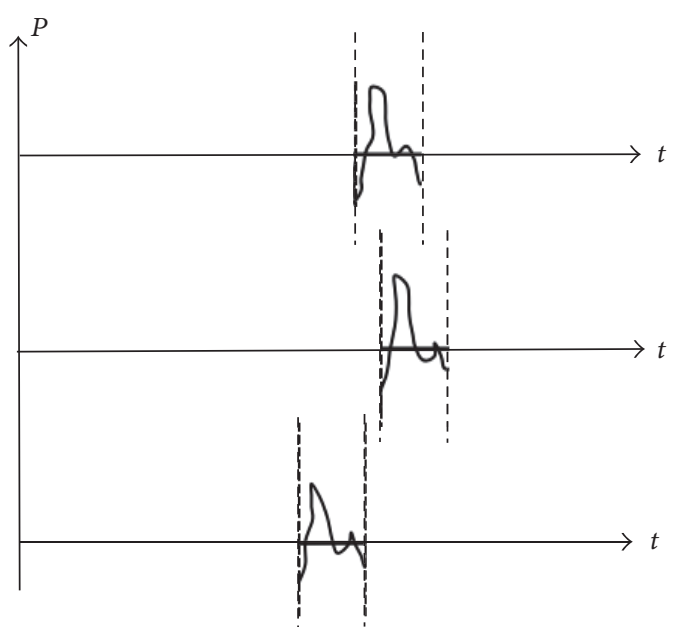

(b)

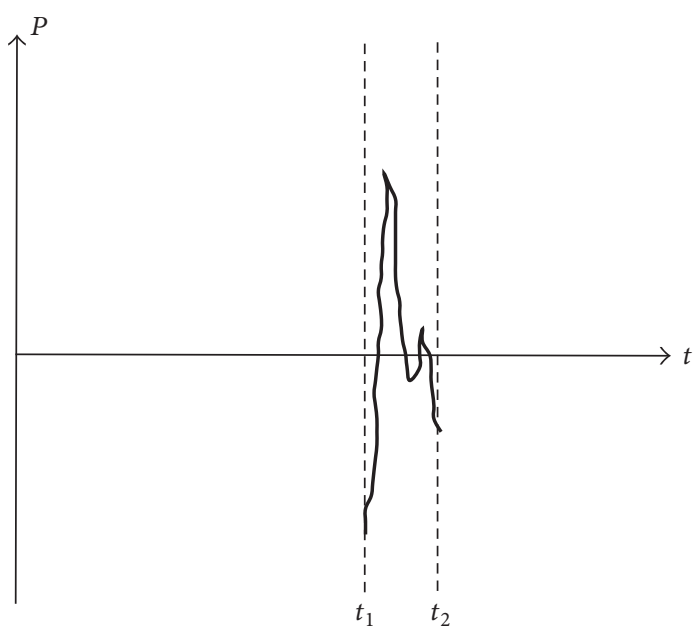

(d)

FIGURE 1: Schematic diagram of beamforming sound field reconstruction.

further improve the accuracy of identification of the sound source.

\section{Acoustical Holography Far-Field Sound Source Identification Method for the Moving Sound Source}

2.1. Short-Time Beamforming. Short beamforming method is based on the "delay accumulate" beamforming signal superimposed principle $[12,13]$. For the reconstruction of the surface at points, the period of its reconstruction $\left[t_{1}, t_{2}\right]$ sound pressure signal within the principle is shown in Figure 1.

First, calculate the time period to be analyzed according to the respective microphone receiving the segment signal period, as shown in Figure 1(a); secondly extracted sound for each signal segments the desired pressure, as shown in Figure 1(b); then, any delay time based on acoustic wave propagation, such as the signal, is $p(t)$, provided that it emits sound waves at time point $s(\varepsilon, \eta)$ to the microphone $i$, the propagation time of $r_{i}(t, \varepsilon, \eta) / c$, and then a signal delay processing as shown in Figure 1(c):

$$
\tilde{p}_{i}=p_{i}\left(t+\frac{r_{i}(t, \varepsilon, \eta)}{c}\right) .
$$

Finally, the superimposed signals of each delay processing, to obtain the reconstruction result of the analysis period, are shown in Figure 1(d).

According to this principle, the sound field characteristic function reconstruction formula at any point $s(\varepsilon, \eta)$ on the reconstruction side $R$ is shown as follows:

$$
\begin{aligned}
& W_{s}(\varepsilon, \eta)=\int_{t_{1}}^{t_{2}} P^{2}(t, \varepsilon, \eta) d t \\
& P(t, \varepsilon, \eta)=\frac{1}{N} \sum_{i=1}^{N} p_{i}\left(t+\frac{r_{i}(t, \varepsilon, \eta)}{c}\right),
\end{aligned}
$$




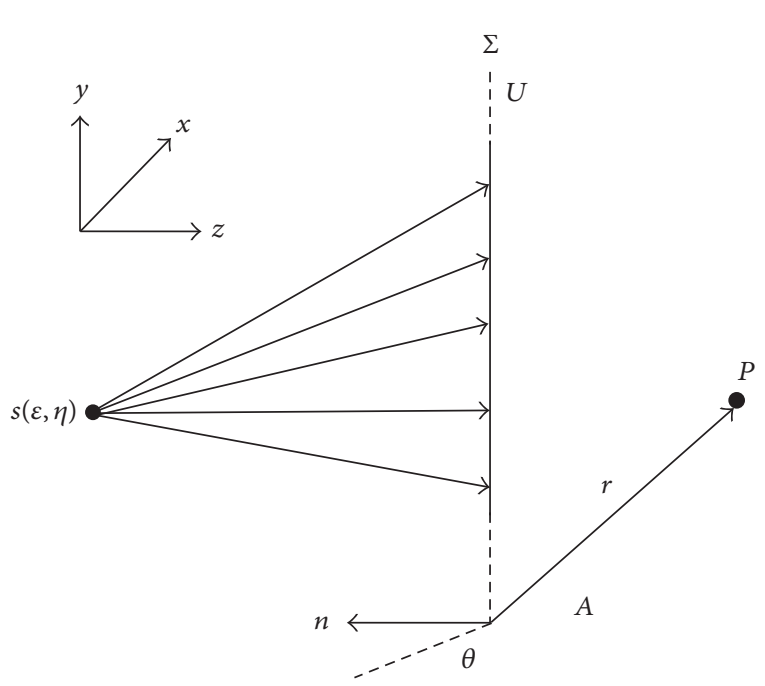

(a)

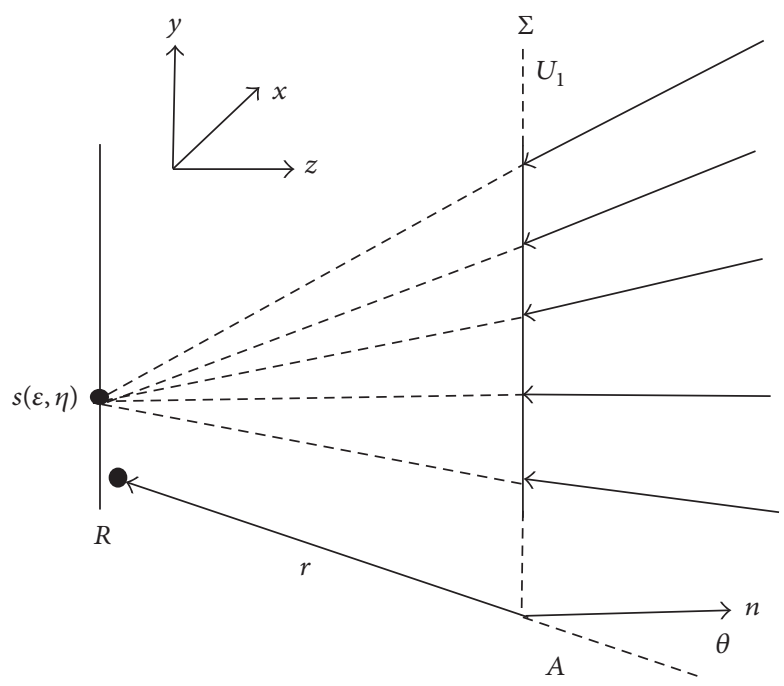

(b)

FIGURE 2: Schematic diffraction of acoustic holography.

where $W_{s}(\varepsilon, \eta)$ is the sound field characteristic function for any point on the surface of the sound source $s(\varepsilon, \eta)$ at time $t$ within $t_{1}-t_{2}, P(t, \varepsilon, \eta)$ is the sound source estimated characteristic function applied of beamforming method, $p_{i}(t)$ is the received sound source pressure signals of $i_{\text {th }}$ microphone at time $t, c$ is the sound velocity, $N$ is the number of microphones, $r_{i}(t, \varepsilon, \eta)$ is the physical distance between the point $s(\varepsilon, \eta)$ in the sound source surface and $i_{\text {th }}$ microphone at time $t$.

Based on this principle, across the entire surface of the sound source, sound field characteristic function of the distribution of the entire surface of the sound source can be obtained inside in time $\left[t_{1}, t_{2}\right]$.

2.2. Far-Field Acoustic Holography Method. NAH (Nearfield Acoustical Holography) method is proposed by Williams et al. $[14,15]$ in the 1980s, and then the method and theory of NAH's applications extend to the far-field conditions. Professor Yang from Tsinghua University has proposed and established the theory of diffraction acoustic holography based on far-field acoustic holography theory. The principle is shown in Figure 2.

In Figure 2, $N$ is the outward normal to the direction of the hologram surface, $r$ is the radius vector, $\theta$ is the angle between the hologram surface $r$ and outward normal direction $n, \Sigma$ is the position in the measuring surface of infinite plane in space, the measurement part of the surface $A$ is $\Sigma$, and $R$ is assumed source point $s$ to the plane, known as the sound source surface.

As it is showed in Figure 2(a) $U$ can be viewed as the point sound source, which assumed that spherical wave at a spatial sound field distribution, $H(x, y, f)$, is $U$. In $\Sigma$ plane component, in the case of $U$ which is known, the wave propagation direction of the space of passive can be calculated by the using of Kirchhoff diffraction integral calculation method. Any point $P$ of the sound field formula is obtained in

$$
U(P)=\frac{1}{4 \pi} \iint_{H}\left[\left(\frac{e^{j k r}}{r}\right) \frac{\partial U}{\partial n}-U \frac{\partial}{\partial n}\left(\frac{e^{j k r}}{r}\right)\right] d \sigma .
$$

Figure 2(b) $S_{1}$ can be viewed as a virtual sound source space. Sound field in the distribution in $S_{1}$, a sonic converge in $S$, spherical wave, and $S$ sent out actually mutually conjugate wave. According to the principle of acoustic holography reconstruction as shown in (4) it is obtained based on the principle of conjugate wave convergence:

$$
\begin{gathered}
U(\varepsilon, \eta, f)=\frac{C k}{j} \iint_{H} H *(x, y, f) \\
\cdot\left[1+\left(1-\frac{1}{j k r}\right) \frac{z_{0}}{r}\right] \frac{e^{-j k r}}{r} d x d y .
\end{gathered}
$$

$k=f / c$ is the wave number, $c$ is the sound velocity, $C$ is a hologram constant, and $r$ is the reconstruction of the surface point $Q(\varepsilon, \eta)$ between the hologram surface points $(x, y)$ distance. Equation (4) is calculated according to the results of a single frequency $f$, continuous several frequencies. Calculating according to the energy superposition can be obtained within a frequency range of the sound pressure amplitude calculation, as shown in

$$
P(\varepsilon, \eta)=\int_{f_{1}}^{f_{2}}|U(\varepsilon, \eta, f)|^{2} d f .
$$

Reconstructing by the above method assumes that the sound pressure of the sound source $Q$ value, as we can see in Figure 3, supposes the point traverse plane of reconstruction 

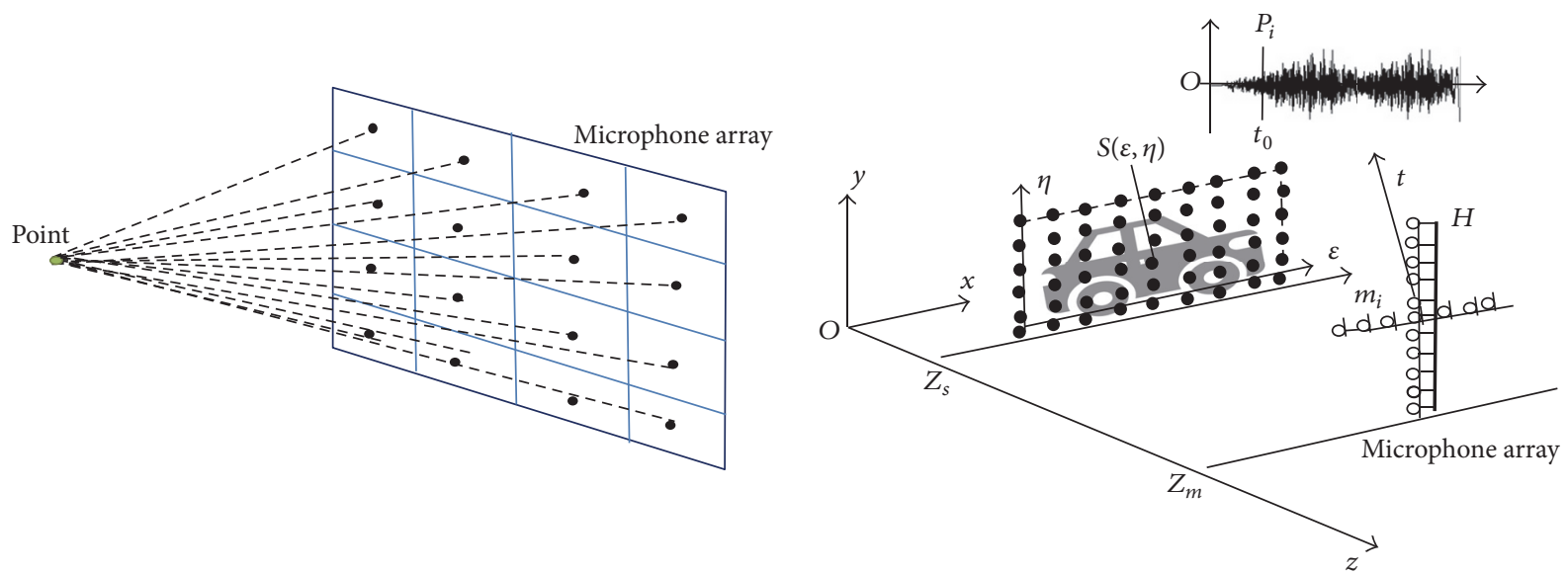

Figure 3: Hologram surface structure.

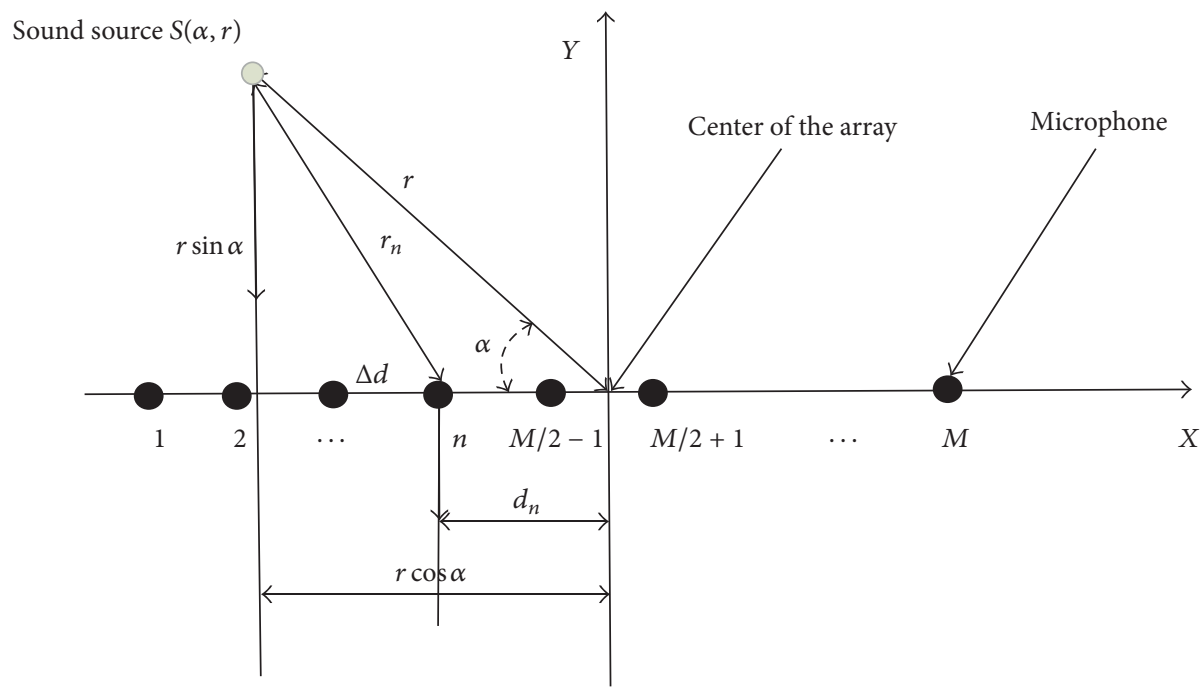

FIGURE 4: Model of uniform linear array.

and repeats this calculation; we can calculate the entire plane of the sound pressure distribution. In actual measurement, the continuous sound pressure points in hologram surface cannot be measured; therefore, (4) is discretized; as shown in Figure 3 for rules discrete arrays can be reconstructed by directly formula, as shown in

$$
\begin{aligned}
U(\varepsilon, \eta, f)= & \frac{C k}{j} \sum_{m=1}^{M} \sum_{n=1}^{N} H^{*}(m, n, f) \\
& \cdot\left[1+\left(1-\frac{1}{j k r}\right) \frac{z_{0}}{r}\right] \frac{e^{-j k r}}{r} d x d y,
\end{aligned}
$$

where $M$ and $N$, respectively, are microphone rows and columns, $H^{*}(m, n, f)$ is the $m$ th row and the $n$th column of the holographic information of the microphone, $r$ is the reconstruction surface points $S(\varepsilon, \eta) m$ th row and the $n$th column from the microphone, and $\Delta x$ and $\Delta y$ are, respectively, microphone spacing and row distance.

\section{Compared Simulation}

3.1. Application in Microphones Array Sound Sources Discrimination. The microphones array's performance mainly reflected the result of spatial resolution and identification precision of source of noises; we used a microphone array of regular arrangement with equal distance as compared with an array of optimization displacement of microphones positions; in both simulations, the number of microphones is the same; but the size of the array may be different in the simulation result. In this paper, we studied the influence of parameters on the performance of the array of arrays. We used a fixed cross X-type microphone array as an example to describe the plane array performance and then analyzes the simulation and discusses the impact of microphones array displacement on the results of the identification. In this paper, we propose a word "fixed array"; it means that the microphones array is to adjust or optimize the $X$ direction than random microphone array displacement. 
TABLE 1: Simulation result of equal distance array and optimization array.

\begin{tabular}{lcc}
\hline & SLR (side lobe ratio) & MLA (main lobe area) \\
\hline Equal distance & 0.2781 & 0.0635 \\
Optimization array & 0.6824 & 0.0310 \\
\hline
\end{tabular}

Performance of the array is mainly reflected in the spatial resolution, such as an array of irregular random arrangement of the microphone as compared with a regular grid array having the same number of microphones, and then how to identify the source of noise at higher frequencies is very important, due to the performance of the microphone array, its array of applications, selection, and design instructive. Figure 4 shows the model of uniform linear array.

This article studies the influence of parameters on the performance of the array of arrays. A fixed cross $\mathrm{X}$-type microphone array as an example describes the plane array performance analysis and process simulation and discusses the impact of microphones arranged in the form of its properties. Based simulated annealing optimization method, the MLA (main lobe area and main lobe energy/sidelobe energy) and SLR (side lobe ratio) were chosen as objective functions, whether it is feasible to get a better result by the means of reducing the numbers of microphones.

3.2. Simulation. Based simulated annealing optimization method, we selected two objective functions, MLA (main lobe area) and SLR (side lobe ratio), which are the parameters of measurement criteria for evaluation of identification results, and we tend to get the value of MLA, the smaller the better, and the value of SLR, the bigger the better, or to get the balance between them. The purpose of the simulation is to compare the microphones array fore-and-aft optimization and then to identify if there would be the possibility of reduction of the numbers of microphones but can achieve a better identification resolution. The simulation result can be seen in Figure 5; in the simulation, the parameters of the sound source are as follows: the speed of sound source is $120 \mathrm{~km} / \mathrm{h}$, the numbers of microphones are 29 in one fixed $\mathrm{X}$ array, and the distance between microphone array and the sound source is $10 \mathrm{~m}$.

Calculation methods of the main lobe area (m2) and the main sidelobe ratio (main lobe energy/sidelobe energy) are shown in Table 1.

As we can get from Figure 5, the result of the simulation is listed in Table 1.

From Table 1, it can be seen that, as a result of optimization array, the value of SLR is 0.6824 , and the value of MLA is 0.0310 , both of the results are better than the results of equal distances microphones array, and the result is in accordance with the objectives and results of optimization we proposed at first. Then we get the 29 optimization microphones' coordinates as shown in Figure 5(b), then we
TABLE 2: Result of optimization microphones array coordinate.

\begin{tabular}{|c|c|c|}
\hline Number of microphones & $X$ & Y \\
\hline 1 & 0 & 0 \\
\hline 2 & 1.9963 & 1.9963 \\
\hline 3 & -1.9963 & 1.9963 \\
\hline 4 & -1.9963 & -1.9963 \\
\hline 5 & 1.9963 & -1.9963 \\
\hline 6 & 2.0000 & 2.0000 \\
\hline 7 & -2.0000 & 2.0000 \\
\hline 8 & -2.0000 & -2.0000 \\
\hline 9 & 2.0000 & -2.0000 \\
\hline 10 & 0.0000 & 0.0000 \\
\hline 11 & 0.0000 & 0.0000 \\
\hline 12 & 0.0000 & 0.0000 \\
\hline 13 & 0.0000 & 0.0000 \\
\hline 14 & 0.0666 & 0.0666 \\
\hline 15 & -0.0666 & 0.0666 \\
\hline 16 & -0.0666 & -0.0666 \\
\hline 17 & 0.0666 & -0.0666 \\
\hline 18 & 1.9371 & 1.9371 \\
\hline 19 & -1.9371 & 1.9371 \\
\hline 20 & -1.9371 & -1.9371 \\
\hline 21 & 1.9371 & -1.9371 \\
\hline 22 & 1.9999 & 1.9999 \\
\hline 23 & -1.9999 & 1.9999 \\
\hline 24 & -1.9999 & -1.9999 \\
\hline 25 & 1.9999 & -1.9999 \\
\hline 26 & 0.0037 & 0.0037 \\
\hline 27 & -0.0037 & 0.0037 \\
\hline 28 & -0.0037 & -0.0037 \\
\hline 29 & 0.0037 & -0.0037 \\
\hline
\end{tabular}

will analyze the ways of easibility of reducing the number of microphones.

The 29 optimization microphones' coordinates are shown in Table 2.

And from Table 2, the coordinate numbers $2-5$ and coordinate numbers $22-25$ are very near to the coordinate [2.0000]; the error is $0.01 \%$ which can be considered as the microphones which are in the same positions; we use coordinate numbers 6-9 to replace the coordinate numbers 2-5 and coordinate numbers $22-25$, and then 8 microphones can be reduced. And coordinate numbers 10-13 are the same as number 1 [0.000], which is in the same positions, and then 4 microphones can be reduced. There were only 17 microphones left. The rest of 17 microphons' simulation is carried out by using the same method, then we get the result of MLA and SLR, and there are no changes in the value of both of them.

In the above simulation, the results show the optimization method which can be achieved to optimize and improve the resolution of the objective function, while achieving the purpose of reducing the number of microphones. 

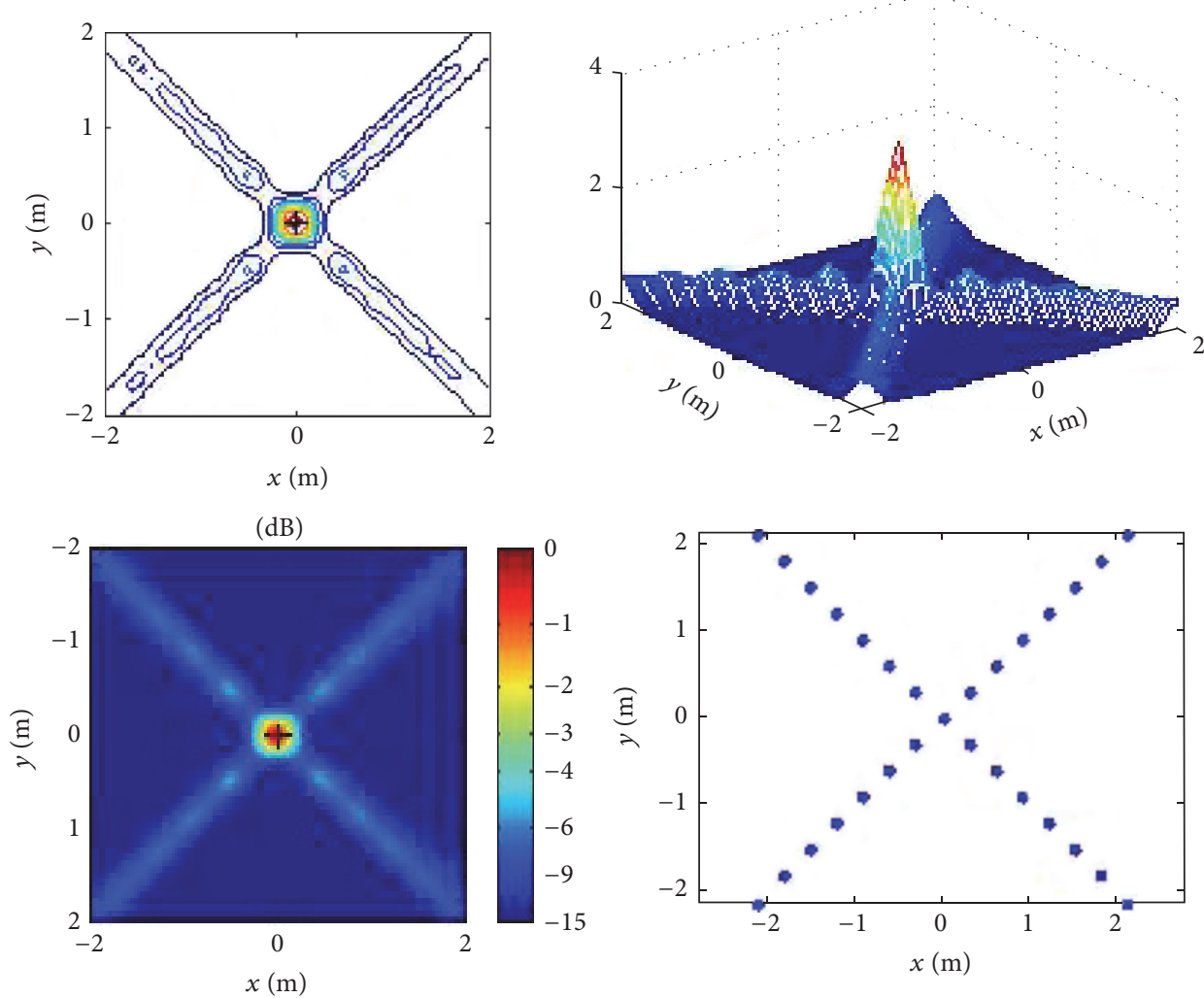

(a)
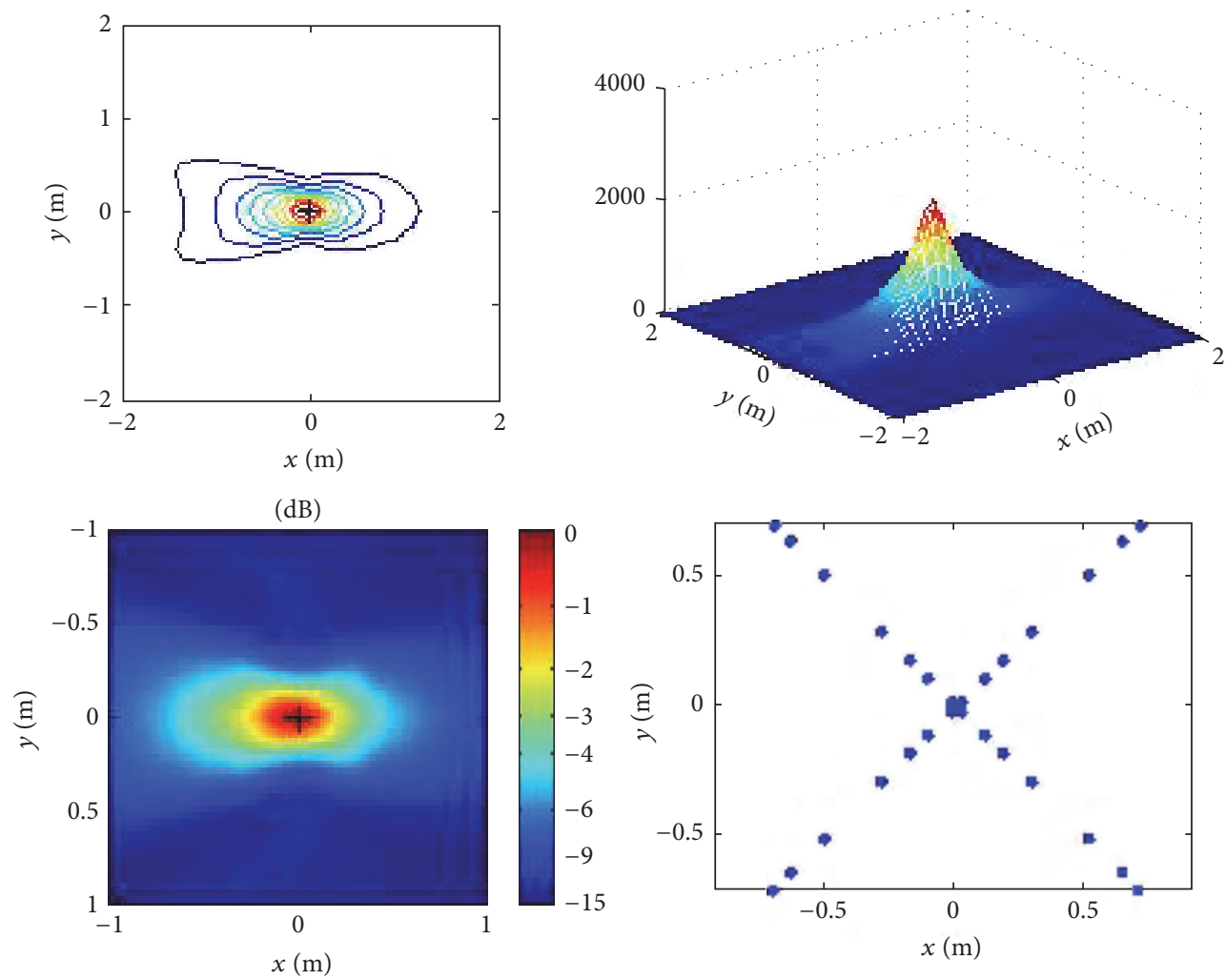

(b)

FIGURE 5: (a) Equal distance array simulation result; (b) optimization array result. 


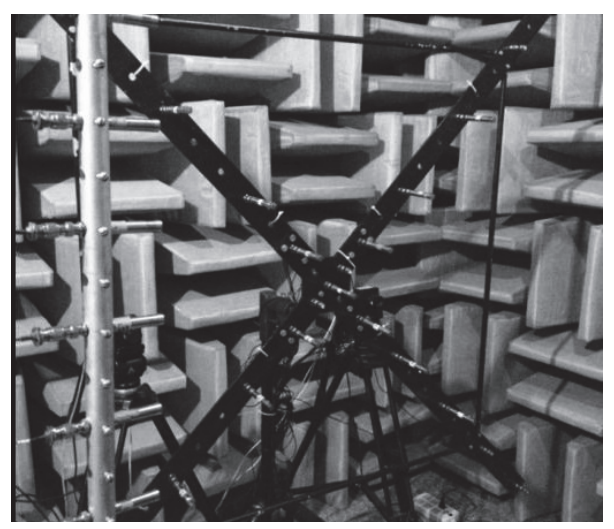

FIGURE 6: The experiment lab and microphone array.
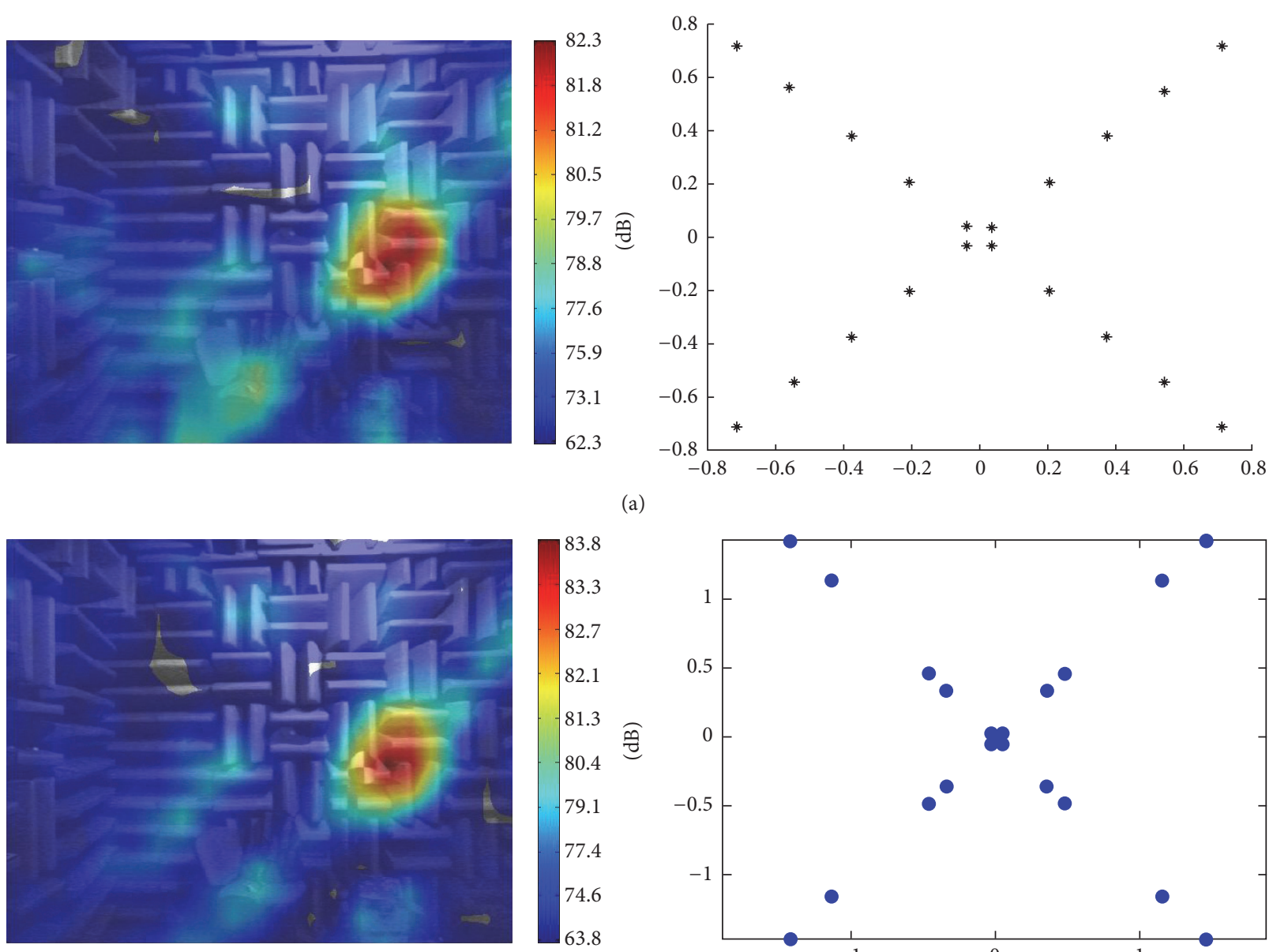

(a)

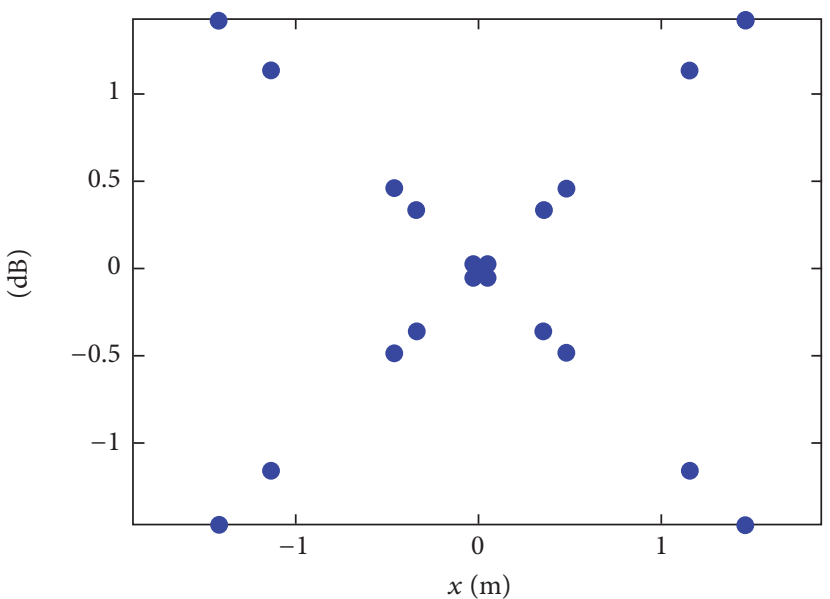

(b)

FIGURE 7: The experiment results of the static speaker in the anechoic room. (a) Result from the acoustic holography method; (b) virtual microphone array. 

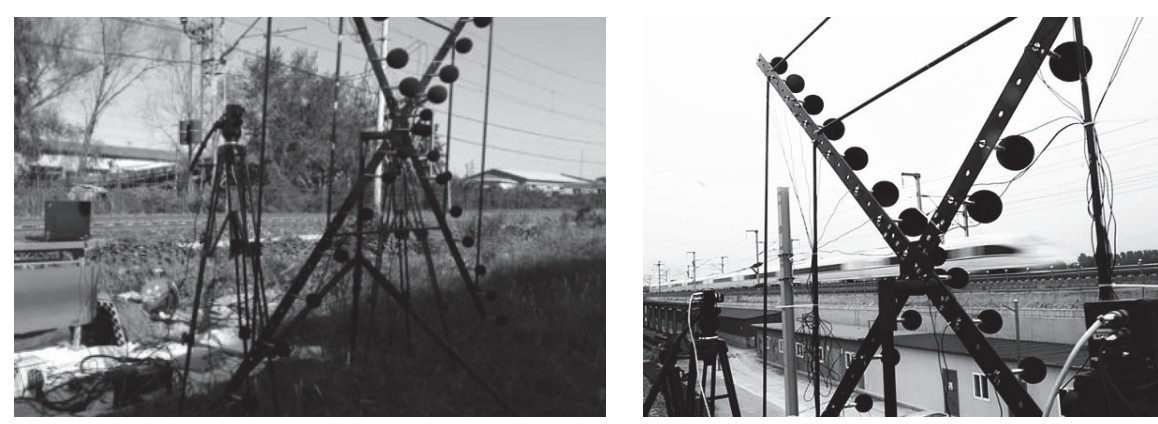

FIGURE 8: The measurement system and testing high speed train.
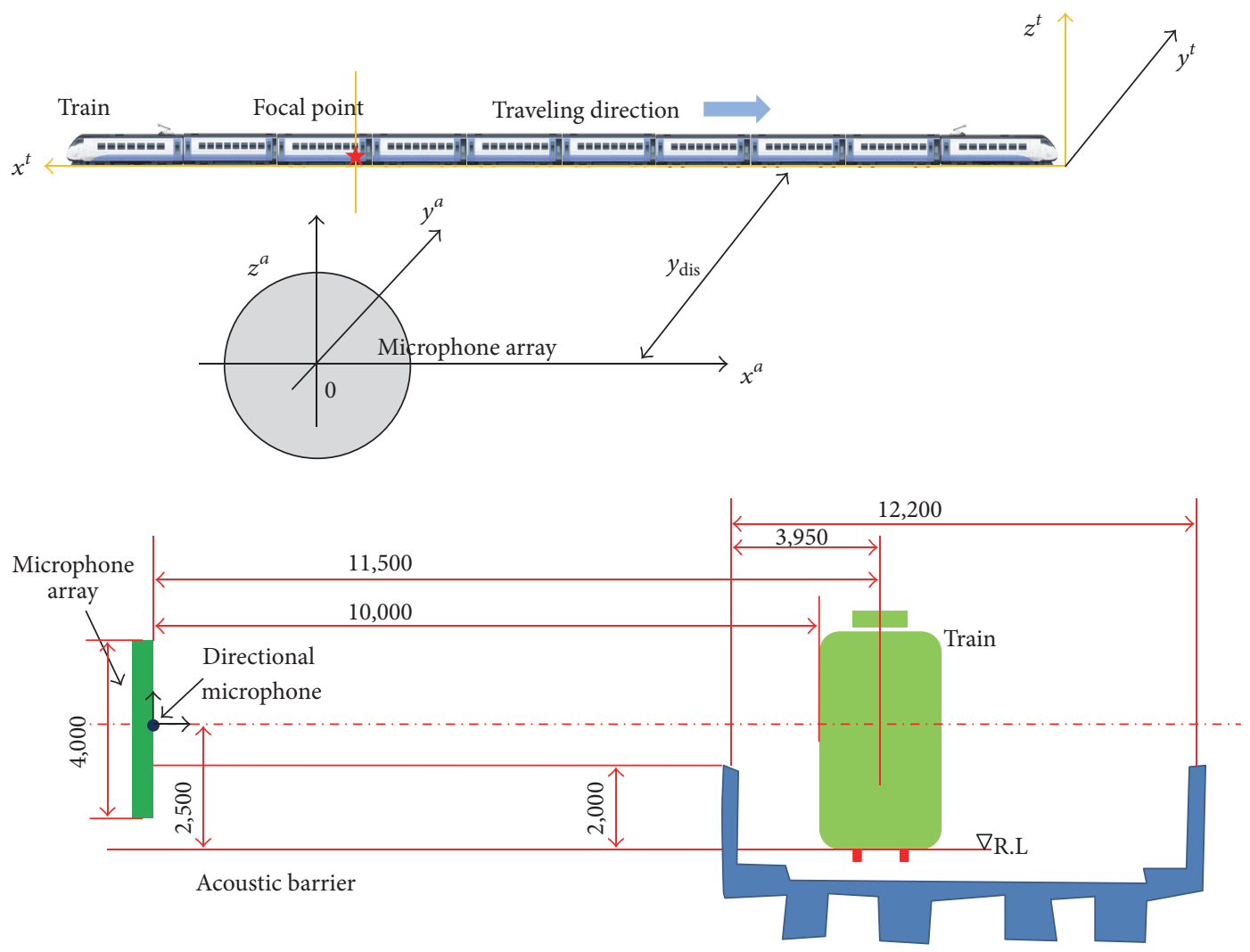

FIGURE 9: The measurement system and testing high speed train.

\section{Validation Experiments}

4.1. Static Sound Sources Experiment. This experiment is done in anechoic chamber. The microphone array is made of 21 microphones. The spacing between each of the microphones is $160 \mathrm{~mm}$. In this experiment, the sound source is a powered loud speaker, and the noise signal is a simple harmonic sound of $1 \mathrm{kHz}$. The experiment lab and experiment device are shown in Figure 6.

As can be seen from Figure 7 and the simulation result in Table 3, comparing both results, there is hardly a change in the value of MLA and SLR (also the result of optimization of MLA (0.0045) is better than equal distance microphones array) and no change in the relative position; to some extent, the result is nearly the same; it is concluded that the 19 microphones nearly have the same solution with 21 microphones.

4.2. High Speed Moving Sound Sources Experiment. The validation experiment is done in circle test ground for high speed train; we choose the high speed train which was 


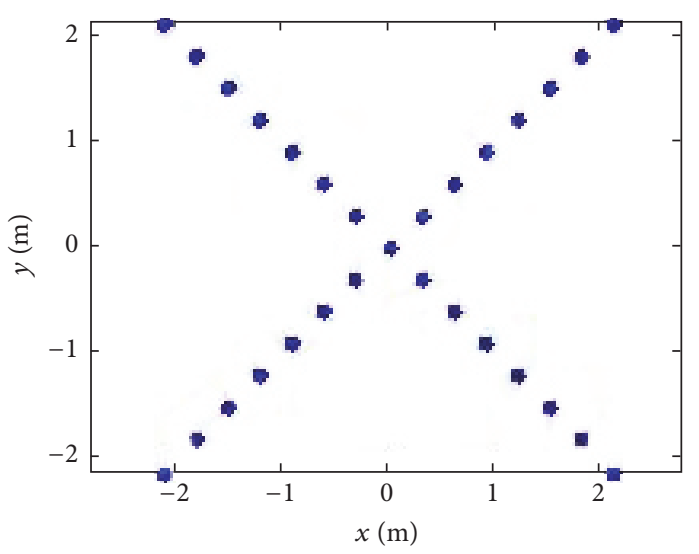

(a)

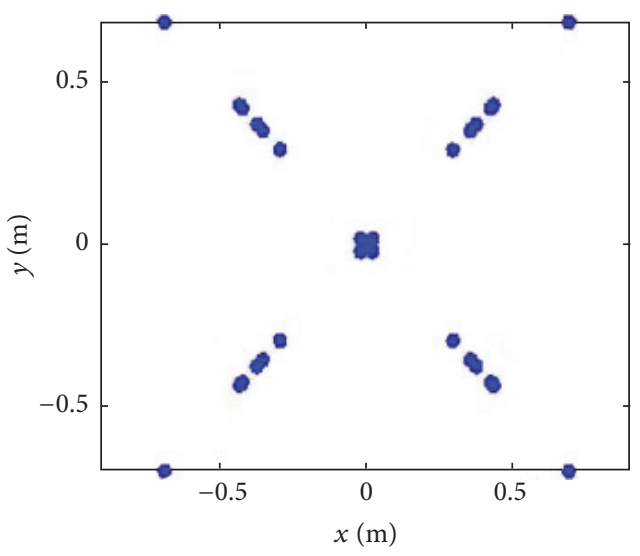

(b)

FIGURE 10: Microphones array. (a) The equal distance; (b) the optimization array.

TABLE 3: Comparison of the results.

\begin{tabular}{lcr}
\hline & \multicolumn{2}{c}{ Equal distance of 21 microphones } \\
(a) & SLR & MLA \\
& 0.5216 & 0.0068 \\
\hline \multicolumn{3}{c}{ Optimization of 19 microphones } \\
(b) & SLR & MLA \\
& 0.4531 & 0.0045 \\
\hline
\end{tabular}

processing test to testify the simulation results. We set the microphones array which is made of 29 microphones and then use the 17 microphones optimization array to process the sound data. The parameters in this experiment are as follows: the sound source is radiated by one high speed train, and the noise signal is a simple harmonic sound of $500 \mathrm{~Hz}-$ $1 \mathrm{kHz}$. The experiment system is shown in Figures 8-10. The main validation experiment device is as follows: conventional signal measurement and analysis equipment, B\&K company's set of high-precision multimicrophone set, 32 sets of high performance acoustic sensors, and an array of developed measurement systems, X-type array.

The experimental conditions are shown in Figure 9. In this experiment, the sound source data analysis frequency is $500-700 \mathrm{~Hz}$, the speed of testing train is $125 \mathrm{~km} / \mathrm{h}$, the compare results are shown in Figure 11, one is the result of identification high speed train sound sources with 29 equal distance microphones' array, and the other is the result of identification high speed train sound sources with optimization microphones array.

The results of experiment are shown in Figure 11 and Table 4 .

As can be seen from the result, both Figure 11 and Table 4, compared with the results, there is hardly change in the value of MLA and SLR (also the result of optimization of MLA $(0.0033)$ is better than equal distance microphones array) and no change in the relative position; to some extent, the result
TABLE 4: Comparison of the results.

\begin{tabular}{lcr}
\hline & \multicolumn{2}{c}{ Equal distance of 29 microphones } \\
(a) & SLR & MLA \\
& 0.6535 & 0.0035 \\
\hline \multicolumn{3}{c}{ Optimization of 17 microphones } \\
(b) & SLR & MLA \\
& 0.6442 & 0.0033 \\
\hline
\end{tabular}

is nearly the same; it is concluded that the 17 microphones nearly have the same solution with 29 microphones.

\section{Conclusion}

In this paper, based on the fixed $\mathrm{X}$ microphones array, select the main SLR (side lobe ratio) and the MLA (main lobe area) as the optimization objective function, by using optimization method to optimize the microphone array. Compare simulation results of equal distance microphones array with optimization array found that reduced the number of optimization microphones; there were no changes in the purpose function value. Also, validation experiment has done to testify the simulation result; it is drawn from the results that the experiment result can be by reducing the number of microphones but does not have changes in the purpose values. So the experiments with limited conditions can be done, as to use fewer microphones to get the similar result. In the next study, it will consider the angle between surface reconstruction of sound pressure and measurement plane by further optimization method.

\section{Competing Interests}

The authors declare that they have no competing interests. 

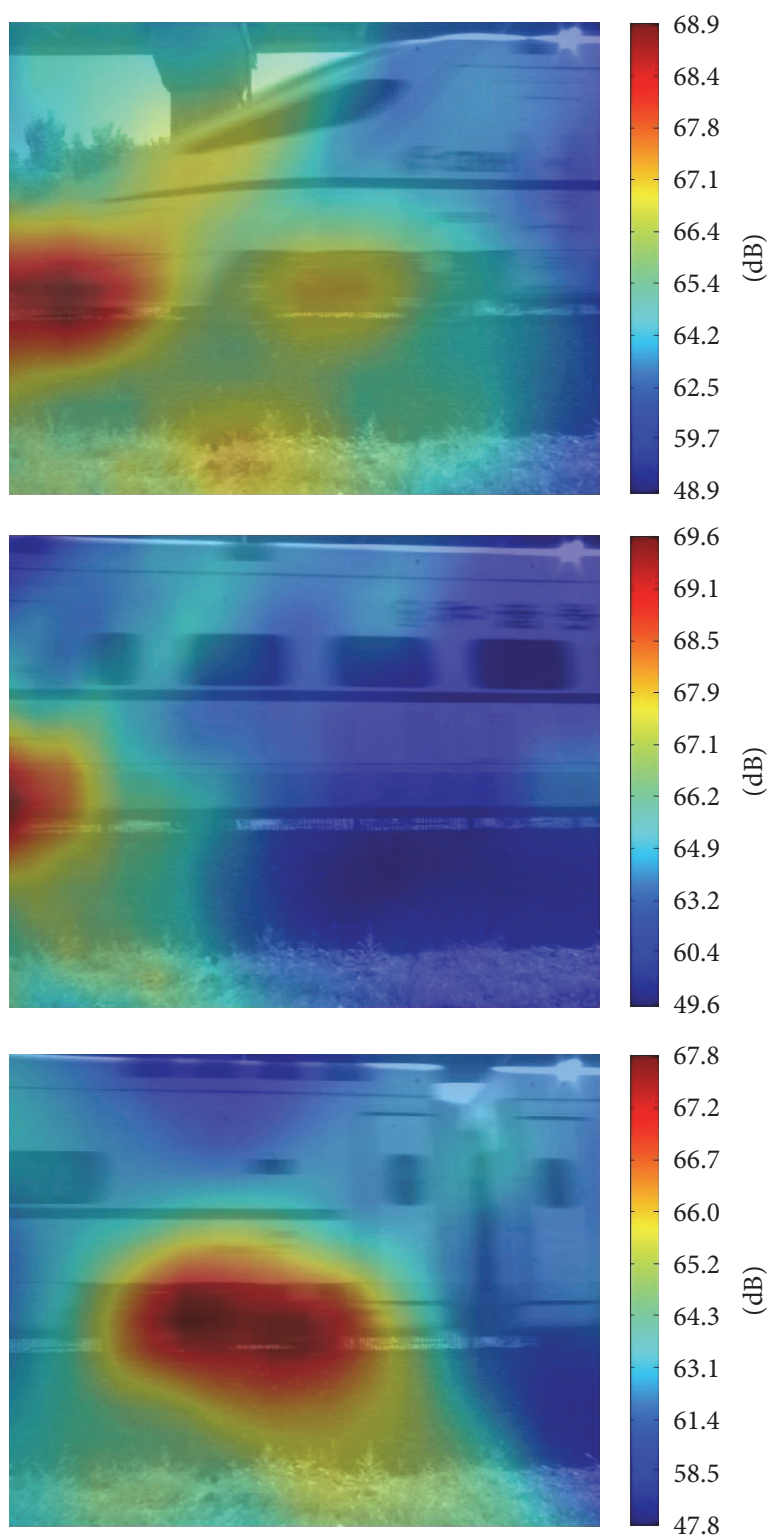

(a)
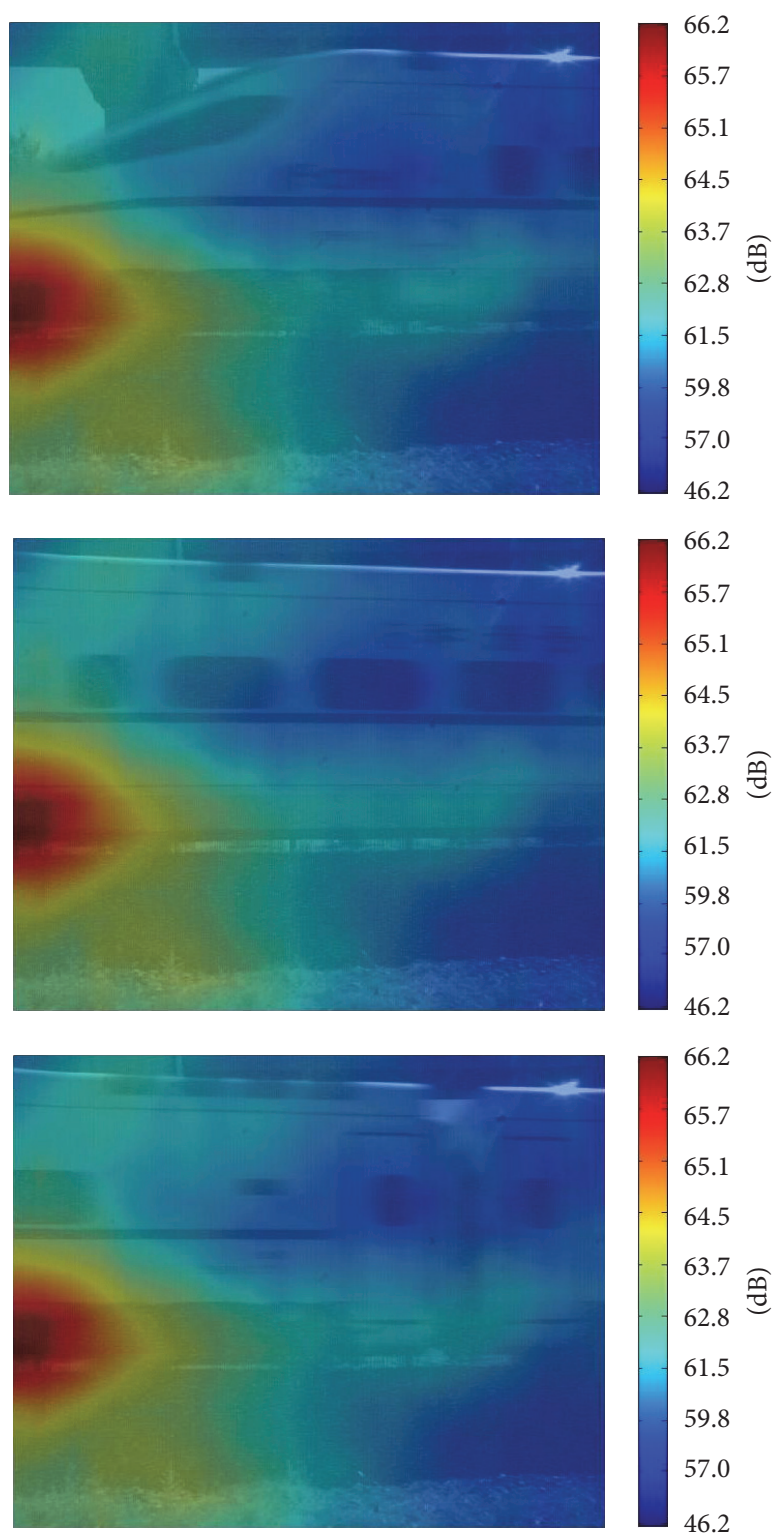

(b)

FIGURE 11: The comparison of validation experiment results of the high speed train with equal distance microphones array (a) and optimization array (b).

\section{References}

[1] H. Pu and J. Weikang, "Application of sound intensity technique in identify the noise sources of maglev train," Journal of Railway Engineering Society, vol. 5, pp. 5-8, 2005.

[2] W. F. King III and D. Bechert, "On the sources of wayside noise generated by high-speed trains," Journal of Sound and Vibration, vol. 66, no. 3, pp. 311-332, 1979.

[3] Y. Takano, K. Terada, F. Aizawa, A. Iida, and H. Fujita, "Development of a 2-dimensional microphone array measurement system for noise sources of fast moving vehicles," in Proceedings of the International Congress on Noise Control Engineering (InterNoise '92), pp. 1175-1178, Toronto, Canada, July 1992.

[4] H. Kook, G. B. Moebs, P. Davies, and J. S. Bolton, "Efficient procedure for visualizing the sound field radiated by vehicles during standardized passby tests," Journal of Sound and Vibration, vol. 233, no. 1, pp. 137-156, 2000.

[5] Y. Takano, "Development of visualization system for highspeed noise sources with a microphone array and a visual sensor," in Proceedings of the International Congress on Noise Control Engineering (InterNoise '03), Seogwipo, Republic of Korea, August 2003.

[6] M. Genescà, J. Romeu, T. Pàmies, and A. Sánchez, "Real time aircraft fly-over noise discrimination," Journal of Sound and Vibration, vol. 323, no. 1-2, pp. 112-129, 2009.

[7] S. Gade, J. Hald, and B. Ginn, "Refined beamforming with increased spatial resolution," in Proceedings of the 41st International Congress and Exposition on Noise Control Engineering (INTER-NOISE '12), pp. 3571-3578, New York, NY, USA, August 2012. 
[8] K. Saijyou and H. Uchida, "Data extrapolation method for boundary element method-based near-field acoustical holography," Journal of the Acoustical Society of America, vol. 115, no. 2, pp. 785-796, 2004.

[9] Z. Wang and S. F. Wu, "Helmholtz equation-least-squares method for reconstructing the acoustic pressure field," Journal of the Acoustical Society of America, vol. 102, no. 4, pp. 20202032, 1997.

[10] R. Steiner and A. N. Kaelin, "Sound field reconstruction of moving noise sources by means of acoustical holography," in Proceedings of the InterNoise 1998, Christchurch, New Zealand, November 1998.

[11] S.-H. Park and Y.-H. Kim, "Visualization of pass-by noise by means of moving frame acoustic holography," Journal of the Acoustical Society of America, vol. 110, no. 5, pp. 2326-2339, 2001.

[12] C.-S. Park and Y.-H. Kim, "Time domain visualization using acoustic holography implemented by temporal and spatial complex envelope," Journal of the Acoustical Society of America, vol. 126, no. 4, pp. 1659-1662, 2009.

[13] D. G. Yang, S. F. Zheng, Y. K. Li, X. M. Lian, and X. Y. Jiang, "Research on acoustic holography method for the identification of sound source," Acta Acustica, vol. 26, no. 2, pp. 156-160, 2001.

[14] Y. Diange, Z. Sifa, L. Bing, L. Keqiang, and L. Xiaomin, "Video visualization for moving sound sources based on binoculars stereo and acoustic holography," Chinese Journal of Acoustics, vol. 30, no. 2, pp. 203-213, 2011.

[15] D. Yang, Z. Wang, B. Li, Y. Luo, and X. Lian, "Quantitative measurement of pass-by noise radiated by vehicles running at high speeds," Journal of Sound and Vibration, vol. 330, no. 7, pp. 1352-1364, 2011. 


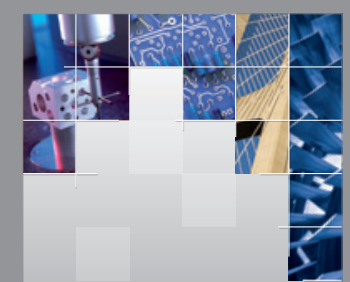

\section{Enfincering}
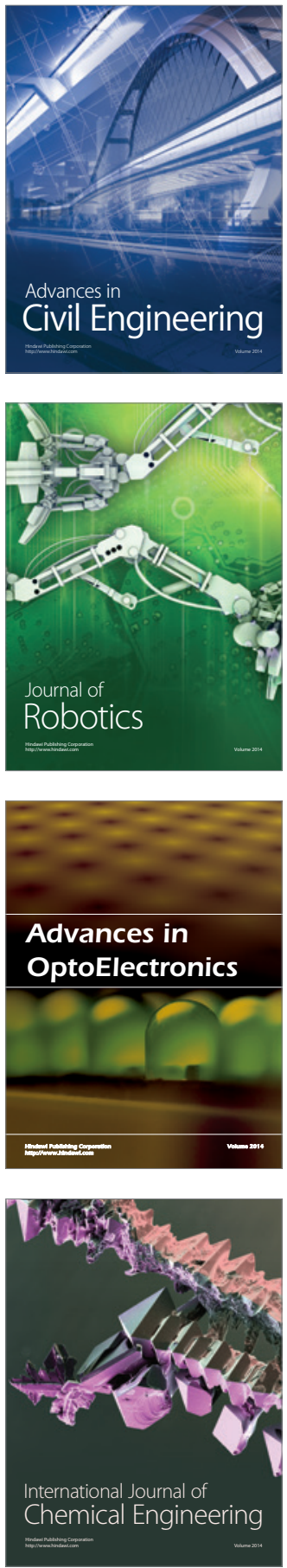

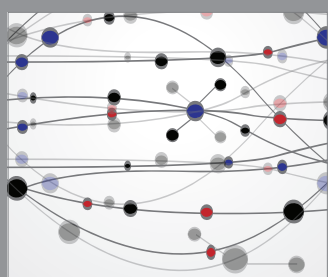

The Scientific World Journal

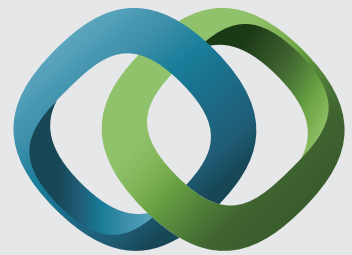

\section{Hindawi}

Submit your manuscripts at

https://www.hindawi.com
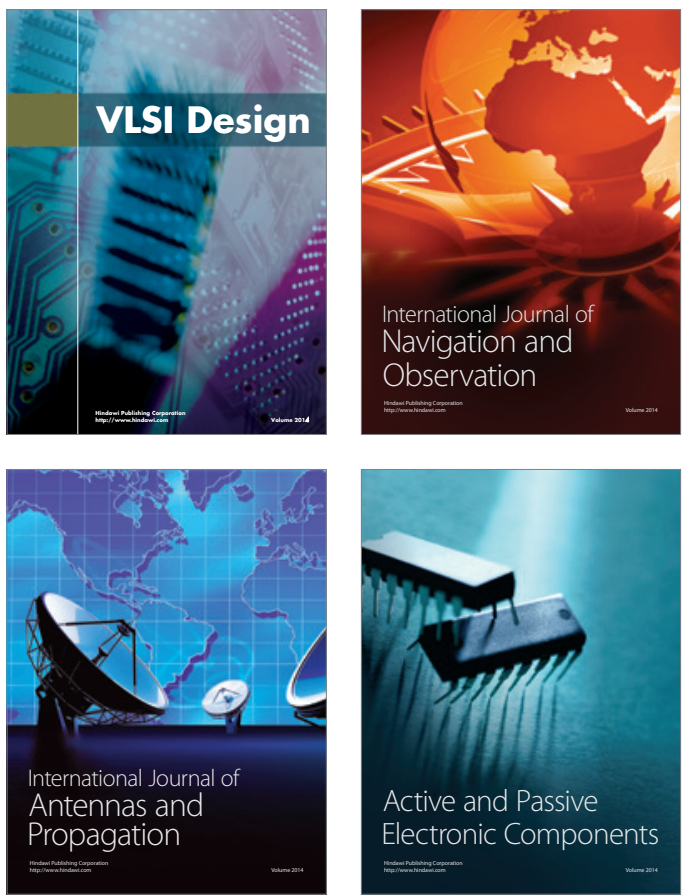
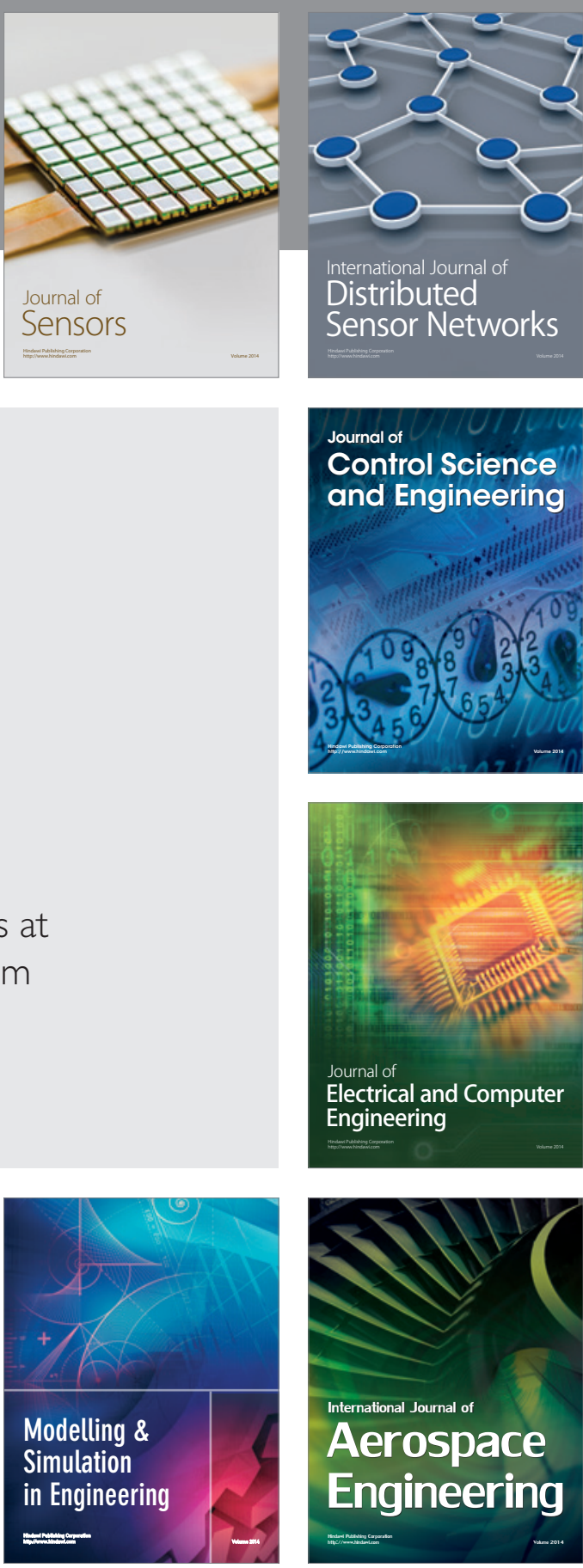

International Journal of

Distributed

Sensor Networks

$-$

Joumal of

Control Science

and Engineering
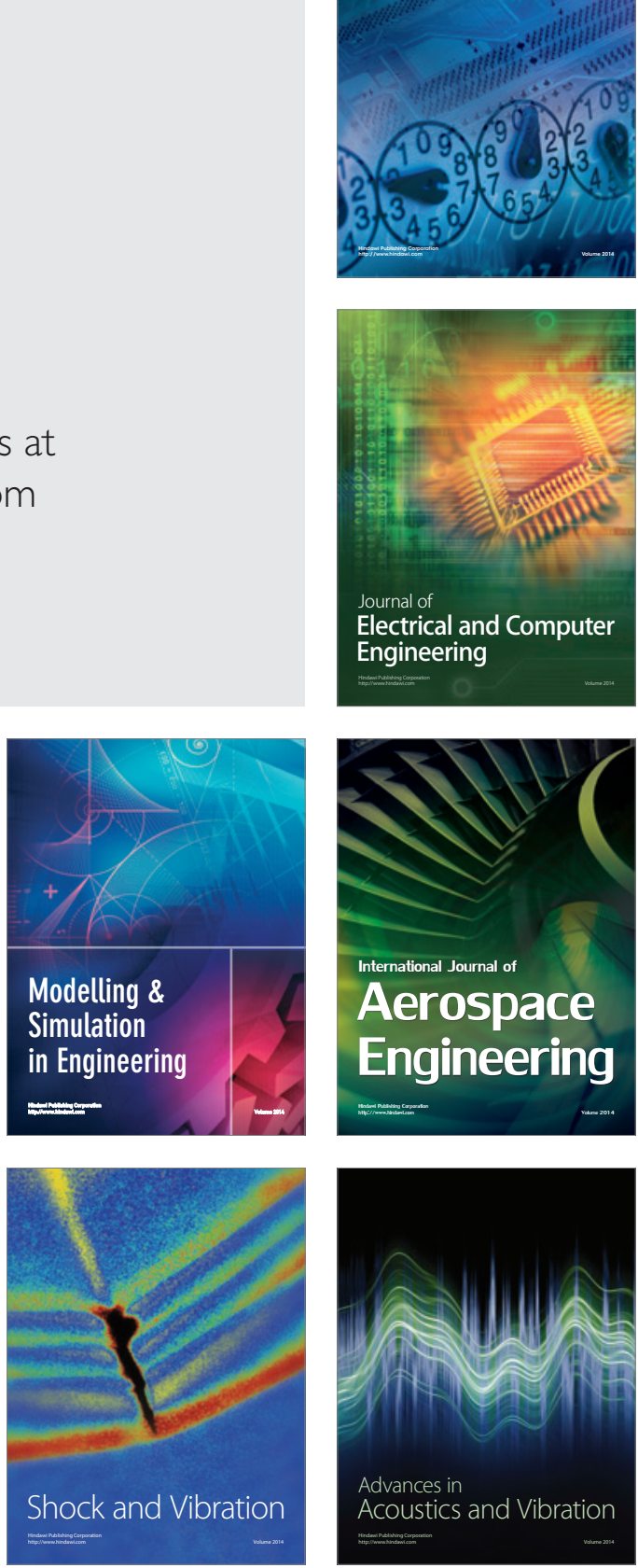